\section{Feedback inhibition of Akt signaling limits the growth of tumors lacking Tsc2}

\author{
Brendan D. Manning, ${ }^{1,5} \mathrm{M}$. Nicole Logsdon, ${ }^{1}$ \\ Alex I. Lipovsky, ${ }^{1}$ Derek Abbott, ${ }^{2,3}$ \\ David J. Kwiatkowski, ${ }^{4}$ and Lewis C. Cantley ${ }^{2}$ \\ ${ }^{1}$ Department of Genetics and Complex Diseases, Harvard \\ School of Public Health; ${ }^{2}$ Department of Systems Biology, \\ Harvard Medical School; Division of Signal Transduction, \\ Department of Medicine, Beth Israel Deaconess Medical \\ Center; ${ }^{3}$ Department of Pathology, ${ }^{4}$ Hematology Division, \\ Department of Medicine, Brigham and Women's Hospital, \\ Harvard Medical School, Boston, Massachusetts 02115, USA
}

The PTEN and TSC2 tumor suppressors inhibit mammalian target of rapamycin (mTOR) signaling and are defective in distinct hamartoma syndromes. Using mouse genetics, we find that Pten and Tsc2 act synergistically to suppress the severity of a subset of tumors specific to loss of each of these genes. Interestingly, we find that the slow-growing tumors specific to $\mathrm{Tsc2}^{+/-}$ mice exhibit defects in signaling downstream of Akt. However, Pten haploinsufficiency restores Akt signaling in these tumors and dramatically enhances their severity. This study demonstrates that attenuation of the PI3K-Akt pathway in tumors lacking TSC2 contributes to their benign nature.

Supplemental material is available at http://www.genesdev.org.

Received March 11, 2005; revised version accepted June 7, 2005.

Growth factor activation of phosphoinositide 3-kinase (PI3K) stimulates cell growth, proliferation, and survival, in large part, through Akt-directed phosphorylation and inhibition of downstream substrates, such as members of the forkhead box $\mathrm{O}$ (FOXO) family of transcription factors, which act to block these processes. The PI3KAkt pathway is also important for growth-factor stimulation of mammalian target of rapamycin (mTOR) signaling (Fingar and Blenis 2004). The primary mechanism by which Akt activates mTOR signaling appears to be through direct phosphorylation and inhibition of TSC2 (also known as tuberin) (Inoki et al. 2002; Manning et al. 2002; Potter et al. 2002; McManus et al. 2004). Interestingly, within this PI3K-Akt-mTOR pathway are two different tumor suppressors, PTEN and TSC2, that function to block growth factor-mediated activation of mTOR (Supplementary Fig. 1A).

The PTEN and TSC2 tumor suppressor genes are both mutated in autosomal dominant hamartomatous tumor

[Keywords: Cowden disease; FOXO; $\mathrm{PKB}$; forkhead; hamartoma syndromes; tuberous sclerosis complex]

${ }^{5}$ Corresponding author.

E-MAIL bmanning@hsph.harvard.edu; FAX (617) 432-5236.

Article published online ahead of print. Article and publication date are at http://www.genesdev.org/cgi/doi/10.1101/gad.1314605. syndromes (Cantley and Neel 1999; Kwiatkowski 2003). Germline mutations in PTEN cause Cowden disease and related hamartoma syndromes, and PTEN loss is also common in a large number of human cancers (Li et al. 1997; Cantley and Neel 1999; Eng 2003). PTEN reduces intracellular levels of PI3K lipid products, thereby inhibiting Akt activation. In cells lacking PTEN, Akt is constitutively active (Stambolic et al. 1998; Sun et al. 1999), and there is a corresponding increase in TSC2 phosphorylation and mTOR signaling, even in the absence of growth factors (Manning et al. 2002). Therefore, it appears that loss of the PTEN tumor suppressor can lead to inactivation of the TSC2 tumor suppressor and subsequent elevation of basal mTOR signaling, suggesting that this might contribute to tumorigenesis resulting from PTEN mutations. Consistent with this notion, $P T E N^{-1-}$ tumors and cell lines, like those expressing oncogenic PI3K or Akt (Aoki et al. 2001; Wendel et al. 2004), are unusually sensitive to mTOR inhibitors (Neshat et al. 2001; Podsypanina et al. 2001). Inactivating mutations in the TSC2 gene or the TSC1 gene, which encodes a TSC2-binding protein, cause the hamartoma syndrome tuberous sclerosis complex (TSC) (Kwiatkowski 2003). Like loss of PTEN, disruption of TSC1 or TSC2 gives rise to cells and tumors with elevated mTOR signaling (Goncharova et al. 2002; Kwiatkowski et al. 2002; El-Hashemite et al. 2003), and rodent models of TSC develop tumors that are highly sensitive to rapamycin and its analogs (Kenerson et al. 2002; Lee et al. 2005). However, unlike PTEN, TSC2 mutations have not been found in sporadic cancers, and while Cowden patients are predisposed to a large number of cancers (Li et al. 1997; Cantley and Neel 1999; Eng 2003), the development of malignancy is very rare in TSC (Kwiatkowski 2003). Therefore, despite these two tumor suppressors acting to inhibit the same pathway and their loss similarly leading to mTOR-dependent tumorigenesis, the malignancy potential of tumors lacking either PTEN or TSC2 differs greatly.

In this study, we examine the genetic relationship between the mammalian Tsc2 and Pten tumor suppressor genes by crossing $\mathrm{Tsc}^{+/-}$mice with $\mathrm{Pten}^{+/-}$mice. We find that these genes act synergistically to suppress a subset of phenotypes specific to each single-heterozygous line. However, the effects on underlying signaling pathways vary between different tumor types. Our data strongly support the hypothesis that attenuation of Akt signaling in TSC-related tumors limits their severity. These findings help explain the large phenotypic differences between Cowden and TSC patients and the apparent absence of TSC2 mutations in sporadic cancers.

\section{Results and Discussion}

It has recently been demonstrated that homozygous loss of Tsc1 or Tsc2 in mouse embryonic fibroblasts (MEFs) and in Drosophila results in a strong inhibition of growth factor-stimulated PI3K signaling (Radimerski et al. 2002; Zhang et al. 2003; Harrington et al. 2004; Shah et al. 2004; for review, see Manning 2004). We find that MEFs lacking Tsc2, unlike their wild-type counterparts, are unresponsive to serum, IGF1, and PDGF for Akt activation and are, likewise, defective in downstream signaling to the Akt targets FOXO1 and FOXO3a (Supple- 
mentary Fig. 1B). Furthermore, FOXO3a protein levels are greatly increased in the Tsc2 $2^{-/}$MEFs, perhaps indicative of a lack of proteasomal degradation previously shown to be stimulated by Akt-mediated phosphorylation (Plas and Thompson 2003). These cell culture experiments raise the intriguing possibility that the benign nature and limited proliferative capacity of TSC tumors, relative to those displaying loss of PTEN, might result from attenuation of Akt signaling. However, whether this negative-feedback loop is active in vivo and affects the behavior of tumors lacking TSC gene function has not been explored previously.

Homozygous loss of either Tsc2 or Pten in mice leads to embryonic lethality (DiCristofano et al. 1998; Suzuki et al. 1998; Kobayashi et al. 1999; Onda et al. 1999; Podsypanina et al. 1999). However, the corresponding heterozygous mice are viable and develop a variety of different neoplasias with nonoverlapping spectra. We assessed the survival of a cohort of 248 offspring (29 litters) from crosses between $\mathrm{Tsc}^{+/-}$and $\mathrm{Pten}^{+/-}$mice over the course of 1 yr (Fig. 1a). No wild-type or $\mathrm{Tsc}^{+/-}$mice from this group died before 1 yr of age. However, there was a reduced survival rate in both the $\mathrm{Pten}^{+/-}$and $\mathrm{Tsc}^{+/-}$ Pten $^{+/-}$mice, with $58 \%$ (40 of 69) and $86 \%$ (43 of 50), respectively, dying within $1 \mathrm{yr}$, and $\mathrm{Tsc}^{+/-}$Pten $^{+/-}$mice exhibited significantly reduced survival compared with Pten $^{+-}$mice $(P=0.0003)$. The reduced survival of $\mathrm{Tsc2}^{+/-} \mathrm{Pten}^{+/-}$mice relative to $\mathrm{Pten}^{+/-}$mice is predominantly due to a decrease in male viability during this interval (Supplementary Fig. 2).

We performed full necropsies on all possible Pten $^{+/-}$ and $\mathrm{Tsc}^{+/-} \mathrm{Pten}^{+/-}$mice that died during the 1-yr survival study (Supplementary Table 1). We detected most of the previously described Pten ${ }^{+/-}$phenotypes in both genotypes, including lymphadenopathy, thyroid adeno-
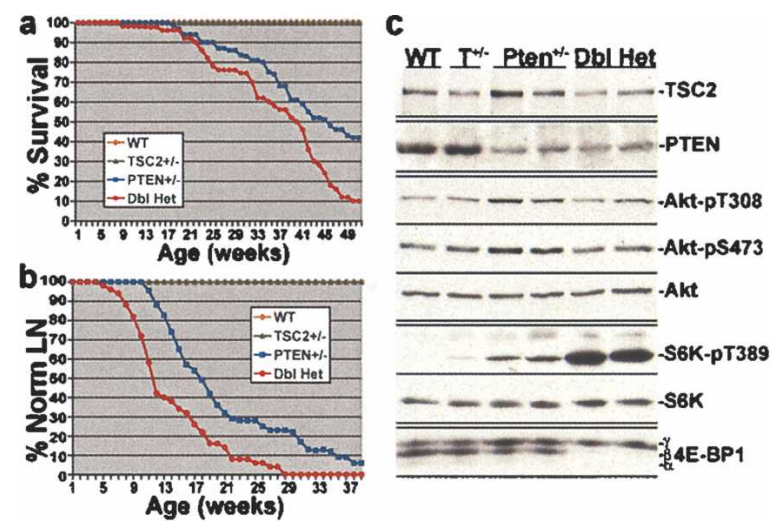

Figure 1. Reduced survival and early development of lymph node hyperplasia in $\mathrm{Tsc}^{+1-}$ Pten $^{+/-}$double-heterozygous mice. (a) Oneyear Kaplan-Meier survival analyses for a cohort of 248 offspring from crosses between $\mathrm{Tsc2}^{+/-}$and $\mathrm{Pten}^{+/-}$mice $(N=70$ wild type [WT], $59 \mathrm{Tsc2}^{+/-}, 69 \mathrm{Pten}^{+/-}$, and $50 \mathrm{Tsc}^{+/-} \mathrm{Pten}^{+/-}$[Dbl Het]). $P=0.0003$ for comparison of Pten $^{+-}$and Dbl Het survival curves. $(b)$ Kaplan-Meier analyses of lymph node hyperplasia detection in the same cohort from a. P $=0.0003$ for comparison of $\mathrm{Pten}^{+/-}$and Dbl Het curves. (c) Presence of TSC2 and PTEN protein and elevated mTOR signaling in the hyperplastic lymph nodes of $\mathrm{TsC2}^{+/-} \mathrm{PTEN}^{+/-}$ mice. Protein extracts from normal (wild-type and $\mathrm{Tsc}^{+/-}\left[\mathrm{T}^{+/-}\right]$) and hyperplastic $\left(\mathrm{Pten}^{+/-}\right.$and Dbl Het) lymph nodes were prepared from six 15-wk-old mice of each genotype as indicated (representative samples for each are shown), normalized for protein concentration (100 $\mu \mathrm{g} / \mathrm{lane})$, and analyzed by immunoblotting, as indicated. mas, pheochromocytomas, gastrointestinal polyps, endometrial hyperplasia, and prostatic hyperplasia (DiCristofano et al. 1998, 1999, 2001; Suzuki et al. 1998; Podsypanina et al. 1999). However, the overall penetrance and severity of these various Pten $^{+/-}$phenotypes were similar in the single- and double-heterozygous mice from this cohort.

As lymph node hyperplasia preceding severe lymphadenopathy is a highly penetrant phenotype in Pten $^{+/-}$ mice (DiCristofano et al. 1999; Podsypanina et al. 1999), we palpated all of the mice in our cohort weekly for 40 wk, starting at weaning $(4 \mathrm{wk})$, to detect this phenotype. Interestingly, hyperplastic lymph nodes were detected, primarily in the axillary and submandibular stations, with an earlier onset in the $\mathrm{Tsc}^{+/-} \mathrm{Pten}^{+/-}$double-heterozygous group (mean $\pm \mathrm{SEM}=14.6 \pm 0.9 \mathrm{wk})$ relative to the $P$ ten $^{+/-}$group $(19.2 \pm 0.8 ; P<0.0001)$ (Fig. 1b). Lymph node hyperplasia was not detected in either the wild-type or $T s c 2^{+/-}$mice within this cohort through $1 \mathrm{yr}$ of age. Consistent with the findings of previous studies (DiCristofano et al. 1999; Podsypanina et al. 1999), the enlarged Pten $^{+/}$and $\mathrm{Tsc}^{+/-}$Pten $^{+/-}$lymph nodes contained expanded populations of both $\mathrm{B}$ and $\mathrm{T}$ lymphocytes, as determined by flow cytometric analyses and immunohistochemistry (IHC) (data not shown), and therefore, appear to be polyclonal in nature. Furthermore, as previously found for Pten $^{+/-}$mice (DiCristofano et al. 1999), PCR (Supplementary Fig. 3A) and immunoblot (Fig. 1c) analyses demonstrate that the lymph node hyperplasia in $\mathrm{TsC2}^{+/-} \mathrm{Pten}^{+/-}$mice is due to haploinsufficiency at both the Pten and Tsc2 loci.

In order to determine the potential mechanism of this enhanced phenotype, signaling downstream of mTOR, including phospho-S6K1 and 4E-BP1 (Fig. 1c) and phospho-S6 (Supplementary Fig. 3B), was examined in lymph node extracts from 15 -wk-old mice of each genotype. Relative to other genotoypes, all hyperplastic $\mathrm{Tsc}^{+/-}$ Pten $^{+/-}$lymph nodes exhibited very high levels of phosphorylated S6K1, S6, and 4E-BP1, while there was only a slight, but reproducible, increase in the $\mathrm{Pten}^{+/-}$samples. Therefore, loss of one copy of each of the Pten and Tsc2 tumor suppressors causes an early onset of lymphoid hyperplasia that coincides with enhanced signaling downstream of mTOR. As both PTEN and TSC2 function to inhibit pathways leading from growth factor receptor stimulation to mTOR signaling (Supplementary Fig. 1A), a combined decrease in their dosage is likely to explain the elevated signaling through this pathway and the corresponding enhanced phenotype. Interestingly, the elevated levels of phospho-Akt observed in the $\mathrm{Pten}^{+/-}$ lymph nodes (DiCristofano et al. 1999) are restored to wild-type levels in the Tsc2 $2^{+/-}$Pten $^{+/-}$lymph nodes (Fig. 1c). This suggests that functional inactivation of the remaining TSC2 protein in these lymphocytes by Pten haploinsufficiency partially activates the feedback inhibition mechanism affecting Akt signaling (Supplementary Fig. 1B). This finding further supports that enhanced mTOR signaling, rather than Akt signaling per se, is responsible for this hyperplasia.

In addition to phenotypes common to $\mathrm{Pten}^{+/-}$mice, we also detected the major tumor types previously described for $\mathrm{Tsc2}^{+/-}$mice (Onda et al. 1999) in the $\mathrm{Tsc2}^{+/-} \mathrm{Pten}^{+/-}$ double-heterozygous mice, including kidney adenomas and cysts, liver hemangiomas, and angiosarcomas (Supplementary Table 1). These tumors were not detected in any wild-type or Pten ${ }^{+/-}$mice. In the survival 
study cohort, angiosarcomas on the extremities (e.g., paw or tail) were detected earlier and with a threefold higher incidence in the $\mathrm{Tsc}^{+/-} \mathrm{Pten}^{+/-}$mice (mean $\pm \mathrm{SEM}=31 \pm 1.0 \mathrm{wk} ; 36 \%, 18$ of 50 ) compared with the $\mathrm{Tsc}^{+/-}$mice $(45 \pm 1.3 \mathrm{wk} ; 11.9 \%$, seven of 59 ; $P=0.003$ in comparison of overall incidence) (Fig. 2a-c). Necropsies also identified an additional three Tsc $^{+/-}$ Pten $^{+-}$mice with internal angiosarcomas (e.g., Fig. 2c). Post-mortem histological analyses of the $\mathrm{Tsc}^{+1-}$ Pten $^{+/-}$ mice from our survival cohort revealed kidney tumors (Fig. 2e) and liver hemangiomas (Fig. 2g) even in mice $<6$ mo of age (Supplementary Table 1). Therefore, in order to compare the onset and occurrence of $\mathrm{Tsc}^{+/-}$phenotypes between single- and double-heterozygous mice, we generated a second cohort from crosses between $\mathrm{Tsc}^{+/-}$and Pten $^{+/-}$mice. We sacrificed 10 animals of each genotype at both $6 \mathrm{mo}$ and $1 \mathrm{yr}$ and analyzed the presence of kidney tumors and liver hemangiomas (Fig. 2a). We found no significant difference in the occurrence of kidney ad-

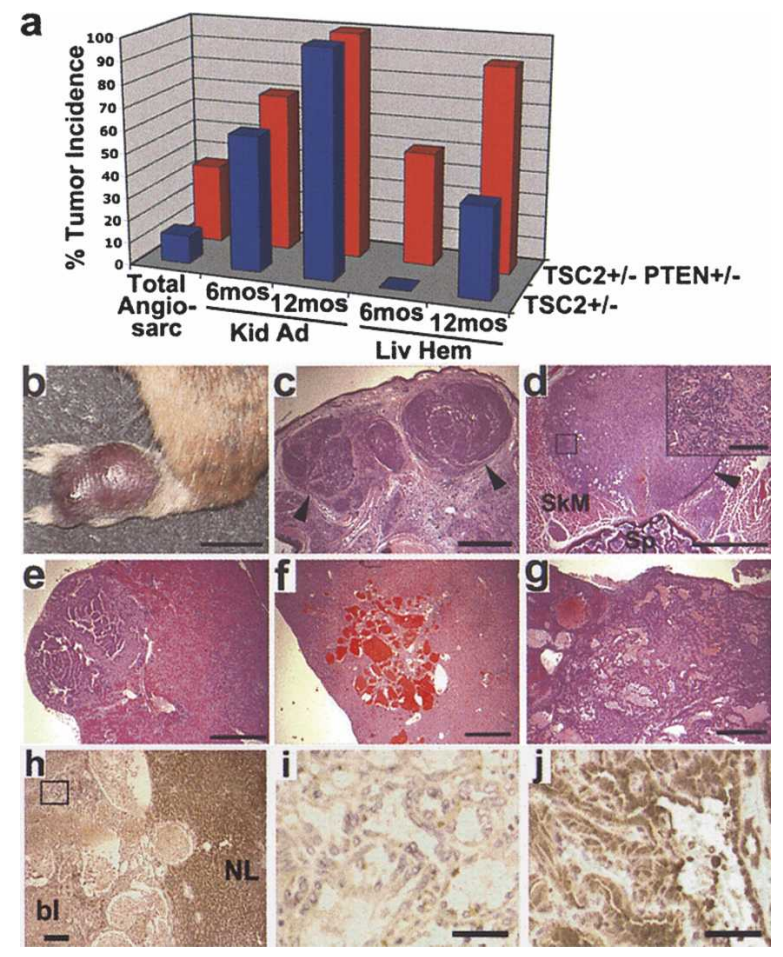

Figure 2. Enhanced angiosarcomas and liver hemangiomas in $\mathrm{TsC2}^{+/-}$Pten $^{+/-}$mice. (a) Summary graph of the incidence of TSCrelated tumors in $\mathrm{Tsc}^{+/-}$and $\mathrm{Tsc}^{+/-} \mathrm{Pten}^{+/-}$mice. See text for details. (b) Macroscopic view of a typical angiosarcoma on the paw of a Tsc2 $2^{+-}$Pten $^{+/-}$mouse at $40 \mathrm{wk}$. (c) A section from the tumor in $b$. (d) An angiosarcoma found adjacent to the spine of a Tsc2 ${ }^{+/-} \mathrm{Pten}^{+/-}$ mouse. (SkM) Skeletal muscle; (Sp) spine. The boxed region is shown at high magnification in the inset. (e) Kidney adenoma from a Tsc2 ${ }^{+/-}$Pten $^{+/-}$mouse at 6 mo. $(f, g)$ Typical liver hemangioma from a 12-mo-old $T s c 2^{+/-}$mouse $(f)$ compared with an example of the lesions found in $\mathrm{Tsc}^{+/-}$Pten $^{+/-}$mice $(g) .(c-g)$ H\&E-stained tumors. $(c-e)$ Arrowheads point to tumor borders. (h) Anti-TSC2 staining of a $\mathrm{Tsc2}^{+/-} \mathrm{Pten}^{+/-}$liver hemangioma and adjacent normal liver (NL). The staining within the vascular lesion is blood (bl). (i) High-magnification view of the box from $h$, demonstrating loss of TSC2 staining within the endothelial cells comprising the liver hemangioma. (j) Maintenance of PTEN expression in a Tsc2 $2^{+/-}$Pten $^{+/-}$liver hemangioma. Bars: $b, 1 \mathrm{~cm} ; c-g, 1 \mathrm{~mm}$; , inset, $100 \mu \mathrm{m} ; h, 200 \mu \mathrm{m} ; i, j$, $40 \mu \mathrm{m}$. enomas and/or cysts between $\mathrm{Tsc}^{+/-}$mice (six of 10) and $\mathrm{Tsc}^{+/-}$Pten $^{+/-}$mice (seven of 10) at 6 mo of age, and this phenotype was $100 \%$ penetrant in both genotypes at 1 yr. The number, size, and severity of these tumors were also indistinguishable in the two genotypes. In contrast, liver hemangiomas were detected with an earlier onset and higher penetrance in the Tsc2 ${ }^{+/-} \mathrm{Pten}^{+/-}$mice (five of 10 at $6 \mathrm{mo}$; nine of 10 at $1 \mathrm{yr}$ ) relative to the $\mathrm{Tsc}^{+-}$mice (zero of 10 at $6 \mathrm{mo}, P=0.03$; four of 10 at $1 \mathrm{yr}, P=0.06$ ). In addition, the overall severity and area of liver affected by hemangiomas in double-heterozygous mice were greatly increased over those of $\mathrm{Tsc}^{+/-}$mice (e.g., Fig. 2, cf. $g$ and f). Consistent with this observation, 34\% (17 of 50) of $\mathrm{Tsc2}^{+/-}$Pten $^{+/-}$mice from our initial survival study died as the result of massive bleeding from large, highly vascular liver hemangiomas (mean age $\pm \mathrm{SEM}=37 \pm 1.1 \mathrm{wk})$. However, no Tsc $2^{+/-}$mice in this cohort died before 1 yr of age.

To understand the potential molecular mechanism of the enhanced liver hemangiomas in $\mathrm{Tsc2}^{+/-} \mathrm{Pten}^{+/-}$ mice, we performed IHC analyses on these tumors. Consistent with previous reports of Tsc2 LOH in liver hemangiomas from $\mathrm{Tsc}^{+/-}$mice (Onda et al. 1999), loss of TSC2 expression in $\mathrm{Tsc}^{+/-} \mathrm{Pten}^{+/-}$hemangiomas was detected specifically in the aberrant endothelial cells that comprise these tumors $(N=5$ livers examined and $>15$ total tumors) (Fig. 2h,i). In contrast, PTEN expression is maintained in these tumors (Figs. 2j, 3f; Supplementary Fig. 4A). Therefore, the aggressive liver hemangiomas arising in $\mathrm{TsC2}^{+-} \mathrm{Pten}^{+/-}$mice appear to be due to loss of Tsc2 in the context of Pten haploinsufficiency. We found high levels of pS6 staining in the endothelial cells of liver hemangiomas from both $\mathrm{Tsc}^{+/-}(N=3$ livers and 7 total tumors) (Fig. 3a) and $\mathrm{Tsc}^{+/-} \mathrm{Pten}^{+/-}$mice $(N=3$ livers and $>9$ total tumors analyzed) (Fig. 3b). However, the intensity of staining and percentage of pS6positive cells within any given area of the liver lesions were indistinguishable between these two lines. This finding is consistent with TSC2 being downstream of PTEN for mTOR regulation (Supplementary Fig. 1A), and therefore, the complete loss of TSC2 expression in both genotypes leads to the same high levels of mTOR signaling. Hence, it is unlikely that differences in mTOR activity account for the striking increase in severity of the liver hemangiomas in the double-heterozygous mice relative to the $\mathrm{TsC2}^{+/-}$mice.

As described above, MEFs lacking Tsc gene function exhibit strong attenuation of PI3K and Akt (Manning 2004) and a loss of FOXO regulation downstream of Akt (Supplementary Fig. 1B). In order to determine whether this feedback inhibition exists in TSC tumors, we examined Akt signaling in liver hemangiomas. It is difficult to detect quantitative differences between various tumors using IHC with phospho-specific antibodies, and we were unable to get reproducible IHC staining of activated/phosphorylated Akt in any of our tissue samples. We therefore took advantage of the fact that the FOXO proteins are well characterized to shuttle between the nucleus, when Akt is inactive, and the cytoplasm, when Akt is active (Tran et al. 2003). Interestingly, FOXO1 localization in the aberrant endothelial cells of $\mathrm{Tsc}^{+/-}$ liver hemangiomas was found to be predominantly nuclear (Fig. 3c). This localization was different from nontumor endothelial cells within the same livers, where FOXO1 was localized to the cytoplasm and, to a lesser extent, the nucleus (Supplementary Fig. 4B). In 


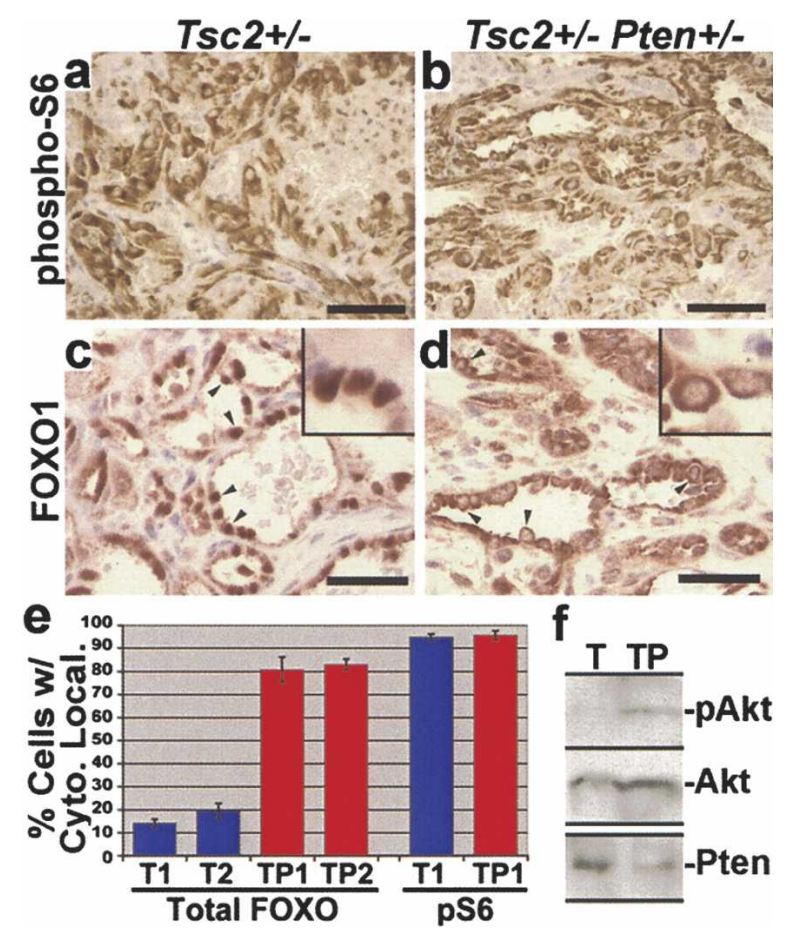

Figure 3. High phospho-S6 levels and differential FOXO1 localization in liver hemangiomas from $\mathrm{Tsc2}^{+/-}$and $\mathrm{Tsc2}^{+/-} \mathrm{Pten}^{+/-}$mice. $(a, b)$ A representative example of high phospho-S6 staining within a $\mathrm{Tsc2}^{+/-}$liver hemangioma $(\mathrm{a})$ and a $\mathrm{Tsc}^{+/-} \mathrm{Pten}^{+/-}$liver hemangioma $(b)$, both from mice aged $12 \mathrm{mo}$. (c-e) Differential localization of FOXO1 in the endothelial cells of $\mathrm{Tsc2}^{+/-}$and $\mathrm{Tsc2}^{+/-} \mathrm{Pten}^{+/-}$liver hemangiomas. A representative example of the nuclear localization of FOXO1 in lesions from $\mathrm{Tsc}^{+/-}$mice $(c)$, in contrast to the predominantly cytoplasmic localization in those from age-matched (12 mo) $\mathrm{Tsc2}^{+-} \mathrm{Pten}^{+/-}$mice (d) (arrowheads point to examples of this localization; insets, $1000 \times$ magnification). Bars: $a-d, 40 \mu \mathrm{m}$. (e) Quantification of endothelial cells from liver hemangiomas showing more cytoplasmic FOXO1 than nuclear. Three hemangiomas per liver for two different $\mathrm{Tsc2}^{+/-}$(T1 and T2) and $\mathrm{Tsc2}^{+-} \mathrm{Pten}^{+/-}$(TP1 and TP2) mice (all aged $12 \mathrm{mo}$ ) were examined. Nuclear/cytoplasmic localization in each endothelial cell positive for FOXO1 staining was scored within these lesions $(>100$ positive cells counted per lesion). Data are graphed as the mean percentage of cells per lesion exhibiting greater cytoplasmic than nuclear FOXO1 staining (error bars indicate standard deviation). $P<0.0001$ for all comparisons between $\mathrm{Pten}^{+-}$and $\mathrm{Tsc2}^{+/-} \mathrm{Pten}^{+-}$data sets. As a control, cytoplasmic phospho-S6 (pS6) staining from adjacent sections of the same lesions was scored. $(f)$ Crude lysates were prepared from liver hemangioma tissue and were analyzed by immunoblotting, as indicated. The total Akt blot was performed after stripping of the phospho-Akt (S473) blot. Representative samples from two of each genotype are shown.

stark contrast to $\mathrm{Tsc}^{+/-}$tumors, the endothelial cells within $\mathrm{TsC}^{+/-}$Pten $^{+/-}$hemangiomas exhibited primarily cytoplasmic FOXO1 localization (Fig. 3d). Quantification of the percentage of cells exhibiting greater cytoplasmic than nuclear FOXO1 staining within hemangiomas from each genotype demonstrated significant differences between Tsc2 $2^{+-}$(mean percent $=14.2 \pm 1.8$ and $19.6 \pm 3.1)$ and Tsc2 $^{+/-}$Pten $^{+/-}$mice $(80.8 \pm 5.4$ and $83 \pm 2.4 ; P<0.0001$ for all data set comparisons between the two genotypes) (Fig. 3e). As a control for the ability to detect cytoplasmic localization in $\mathrm{Tsc}^{+/-}$tumors, cytoplasmic pS6 staining was quantified in adjacent sections from the same tumors and found to be nearly identical in the two genotypes $\left(\mathrm{Tsc}^{+/-}, 94.8 \pm 1.4 ; \mathrm{Tsc}^{+/-} \mathrm{Pten}^{+/-}\right.$, $95.6 \pm 2)$. Finally, we have also observed attenuation of Akt phosphorylation in crude liver hemangioma lysates prepared from $\mathrm{Tsc}^{+/-}$but not $\mathrm{Tsc}^{+/-} \mathrm{Pten}^{+/-}$mice (Fig. 3f). Therefore, while mTOR signaling appears to be the same in liver hemangiomas from single- and doubleheterozygous mice, signaling downstream of Akt is attenuated in the benign tumors of $\mathrm{Tsc}^{+/-}$mice but not in the more aggressive tumors arising in $\mathrm{TsC}^{+/-} \mathrm{Pten}^{+/-}$ mice.

This result demonstrates that loss of Tsc2 expression in tumors triggers a feedback inhibition of Akt signaling, which Pten haploinsufficiency can overcome. In vitro studies on Tsc1-/- and Tsc2 ${ }^{-/-}$MEFs have demonstrated that this negative feedback mechanism blocks receptor tyrosine kinase-mediated activation of PI3K (Zhang et al. 2003; Harrington et al. 2004; Shah et al. 2004). Furthermore, heterozygous loss of Pten has been shown to be sufficient to activate Akt in the absence of receptor stimulation (DiCristofano et al. 1999; Li et al. 2002). Therefore, it seems likely that the TSC-related tumors that develop in the $\mathrm{Tsc}^{+/-} \mathrm{Pten}^{+/-}$mice are more severe due to reactivation of the many proliferation and survival pathways downstream of Akt, which are normally attenuated in absence of Tsc gene function.

In general, our findings are in agreement with a recently completed study by Ma et al. (2005), who also find that $\mathrm{Tsc}^{+/-}$Pten $^{+/-}$mice exhibit reduced survival and that double haploinsufficiency leads to enhanced-Pten ${ }^{+/-}$ phenotypes. However, they find an increase in prostate cancer, which we do not observe, and report no difference in TSC-related phenotypes in their Tsc2 ${ }^{+/-}$Pten $^{+/-}$ mice. These discrepancies are very likely due to strain differences, as studies on both $\mathrm{Pten}^{+/-}$mice and $\mathrm{TsC2}^{+/-}$ mice have detected strain-dependent variations in tumor spectra. This is underscored by the fact that the two TSC-related tumor types that we find to be enhanced in our double-heterozygous mice, angiosarcomas and liver hemangiomas, are not detected at all in the $\mathrm{Tsc}^{+/-}$mice analyzed in this other study. The initial $\mathrm{Tsc}^{+/-}$strains used in the two studies are from different sources (Kobayashi et al. 1999; Onda et al. 1999), and those in the Ma et al. (2005) study, unlike those described here, have not been reported to develop angiosarcomas and require substantial aging $(>18 \mathrm{mo})$ to develop liver hemangiomas (Kobayashi et al. 1999). However, the kidney adenomas that develop in their $\mathrm{Tsc}^{+/-}$mice, as in ours, are not accelerated by Pten haploinsufficiency. It should be noted that, while these kidney adenomas share a location with human TSC tumors, they are not related to the kidney angiomyolipomas (AMLs) most common in TSC patients $(>80 \%)$ (Henske 2005). AMLs more closely resemble the hemangiomas found in our $\mathrm{TsC2}^{+/-}$mice, as both are highly vascular lesions that contain a smooth muscle component and express elevated levels of the estrogen receptor and the HMB45 antigen (El-Hashemite et al. 2005).

Our data demonstrate that feedback inhibition of Akt signaling can limit the growth of these TSC-related tumors, and this is likely to explain the benign nature of tumors arising in TSC patients, relative to those caused by loss of PTEN. These findings also suggest that genetic or physiological events leading to reactivation of Akt, or specific signaling events downstream of Akt, might contribute to the large variability in tumor burden and severity observed in TSC patients. 


\section{Materials and methods}

Mice

The $\mathrm{Pten}^{+/-}$and $\mathrm{Tsc2}^{+/-}$mice used in this study were characterized previously (DiCristofano et al. 1998, 1999, 2001; Onda et al. 1999). The two starting strains were both on a mixed C57BL/6j-129/SvJae background and were expanded by crosses with wild-type $129 /$ SvJae mice, and the offspring were mated to generate the study groups described. PCR analyses of genomic tail DNA were used for genotyping as described previously (DiCristofano et al. 1998; Onda et al. 1999).

\section{Survival curves}

The Kaplan-Meier plot in Figure 1a includes mice that were found dead or were sacrificed for humane reasons due to morbidity. The plot in Figure 1c was generated by weekly examination of mice in the survival cohort for palpable hyperplastic lymph nodes (estimated to be $2-4 \mathrm{~mm}$ in diameter upon detection).

\section{PCR analyses and immunoblotting}

PCR analyses of DNA isolated from normal and hyperplastic lymph nodes, as well as corresponding tail DNA from the same mice, were performed using previously described methods for Tsc2 and Pten genotyping (DiCristofano et al. 1998; Onda et al. 1999). Protein extracts from these lymph nodes and from liver hemangiomas were prepared and analyzed by immunoblotting as described previously (Manning et al. 2002; Shaw et al. 2004). Antibodies used for these studies include the C-20 tuberin (TSC2) antibody from Santa Cruz Biotechnology and PTEN, phospho-Akt (T308), phospho-Akt (S473), Akt, phospho-S6K1 (T389), 4EBP1, phospho-ribosomal S6 (Ser235/236), and eIF4E antibodies from Cell Signaling Technologies.

\section{Histology and IHC}

For full necropsies, mice were submerged in Bouin's fixative solution following dissection to expose internal organs and brain tissue. To determine pathological status, all tissues were examined macroscopically and microscopically following paraffin embedding, sectioning, and H\&E staining. IHC using the N-19 tuberin (TSC2) antibody from Santa Cruz Biotechnology was done on Bouin's-fixed, paraffin-embedded tissue sections. All other IHCs, including PTEN/MMAC1 Ab-2 from NeoMarkers, Inc., and FKHR (FOXO1) and phospho-ribosomal S6 (Ser235/236) antibodies from Cell Signaling Technologies, were done on $10 \%$ formalinfixed, paraffin-embedded tissue sections. IHC slides were prepared according to the antibody manufacturer's instructions following heat-induced antigen retrieval and were detected using Evision+ (DakoCytomation).

\section{Statistical analyses}

$P$-values for Kaplan-Meier plots were determined by Mantel-Cox logrank tests. The rates of occurrence of specific tumor types were compared using the two-sample $t$-test with a two-tailed distribution for samples where $N>20$ and the Fisher's exact test for those where $N<20$.

\section{Acknowledgments}

We are indebted to Dr. P.P. Pandolfi for generously providing the Pten ${ }^{+/-}$ mice and for sharing unpublished data; J. Luo, Dr. N. El-Hashemite, and Dr. L. Pao for technical assistance and advice; Dr. R. Bronson for assistance with histopathology; and Dr. R. Shaw for critical comments on the manuscript. This work was supported by grants from the American Cancer Society and Leukemia and Lymphoma Society awarded to B.D.M, NIH grant numbers NS31535 and CA89021 awarded to D.J.K. and L.C.C., respectively, and a Rothberg Courage Award from the Tuberous Sclerosis Alliance awarded to B.D.M., D.J.K., and L.C.C.

\section{References}

Aoki, M., Blazek, E., and Vogt, P.K. 2001. A role of the kinase mTOR in cellular transformation induced by the oncoproteins P3k and Akt. Proc. Natl. Acad. Sci. 98: 136-141.

Cantley, L.C. and Neel, B.G. 1999. New insights into tumor suppression: PTEN suppresses tumor formation by restraining the phosphoinositide 3-kinase/AKT pathway. Proc. Natl. Acad. Sci. 96: 4240-4245.

DiCristofano, A., Pesce, B., Cordon-Cardo, C., and Pandolfi, P.P. 1998.
Pten is essential for embryonic development and tumour suppression. Nat. Genet. 19: 348-355.

DiCristofano, A., Kotsi, P., Peng, Y.F., Cordon-Cardo, C., Elkon, K.B., and Pandolfi, P.P. 1999. Impaired Fas response and autoimmunity in Pten $^{+/-}$mice. Science 285: 2122-2125.

DiCristofano, A., DeAcetis, M., Koff, A., Cordon-Cardo, C., and Pandolfi, P.P. 2001. Pten and p27KIP1 cooperate in prostate cancer tumor suppression in the mouse. Nat. Genet. 27: 222-224.

El-Hashemite, N., Zhang, H., Henske, E.P., and Kwiatkowski, D.J. 2003. Mutation in TSC2 and activation of mammalian target of rapamycin signalling pathway in renal angiomyolipoma. Lancet 361: 1348-1349.

El-Hashemite, N., Walker, V., and Kwiatkowski, D.J. 2005. Estrogen enhances whereas tamoxifen retards development of Tsc mouse liver hemangioma: A tumor related to renal angiomyolipoma and pulmonary lymphangioleiomyomatosis. Cancer Res. 65: 2474-2481.

Eng, C. 2003. PTEN: One gene, many syndromes. Hum. Mutat. 22: 183198.

Fingar, D.C. and Blenis, J. 2004. Target of rapamycin (TOR): An integrator of nutrient and growth factor signals and coordinator of cell growth and cell cycle progression. Oncogene 23: 3151-3171.

Goncharova, E.A., Goncharov, D.A., Eszterhas, A., Hunter, D.S., Glassberg, M.K., Yeung, R.S., Walker, C.L., Noonan, D., Kwiatkowski, D.J., Chou, M.M., et al. 2002. Tuberin regulates p70 S6 kinase activation and ribosomal protein S6 phosphorylation: A role for the TSC2 tumor suppressor gene in pulmonary lymphangioleiomyomatosis (LAM). J. Biol. Chem. 277: 30958-30967.

Harrington, L.S., Findlay, G.M., Gray, A., Tolkacheva, T., Wigfield, S., Rebholz, H., Barnett, J., Leslie, N.R., Cheng, S., Shepherd, P.R., et al. 2004. The TSC1-2 tumor suppressor controls insulin-PI3K signaling via regulation of IRS proteins. J. Cell. Biol. 166: 213-223.

Henske, E.P. 2005. Tuberous sclerosis and the kidney: From mesenchyme to epithelium, and beyond. Pediatr. Nephrol. 20: 854-857.

Inoki, K., Li, Y., Zhu, T., Wu, J., and Guan, K.L. 2002. TSC2 is phosphorylated and inhibited by Akt and suppresses mTOR signalling. Nat. Cell. Biol. 4: 648-657.

Kenerson, H.L., Aicher, L.D., True, L.D., and Yeung, R.S. 2002. Activated mammalian target of rapamycin pathway in the pathogenesis of tuberous sclerosis complex renal tumors. Cancer Res. 62: 5645-5650.

Kobayashi, T., Minowa, O., Kuno, J., Mitani, H., Hino, O., and Noda, T. 1999. Renal carcinogenesis, hepatic hemangiomatosis, and embryonic lethality caused by a germ-line Tsc 2 mutation in mice. Cancer Res. 59: 1206-1211.

Kwiatkowski, D.J. 2003. Tuberous sclerosis: From tubers to mTOR. Ann. Hum. Genet. 67: 87-96.

Kwiatkowski, D.J., Zhang, H., Bandura, J.L., Heiberger, K.M., Glogauer, M., El-Hashemite, N., and Onda, H. 2002. A mouse model of TSC1 reveals sex-dependent lethality from liver hemangiomas, and upregulation of p70S6 kinase activity in TSC1 null cells. Hum. Mol. Genet. 11: 525-534.

Lee, L., Sudentas, P., Donohue, B., Asrican, K., Worku, A., Walker, V., Sun, Y., Schmidt, K., Albert, M.S., El-Hashemite, N., et al. 2005. Efficacy of a rapamycin analog (CCI-779) and IFN- $\gamma$ in tuberous sclerosis mouse models. Genes Chromosomes Cancer 42: 213-227.

Li, J., Yen, C., Liaw, D., Podsypanina, K., Bose, S., Wang, S.I., Puc, J., Miliaresis, C., Rodgers, L., McCombie, R., et al. 1997. PTEN, a putative protein tyrosine phosphatase gene mutated in human brain, breast, and prostate cancer. Science 275: 1943-1947.

Li, L., Liu, F., Salmonsen, R.A., Turner, T.K., Litofsky, N.S., Di Cristofano, A., Pandolfi, P.P., Jones, S.N., Recht, L.D., and Ross, A.H. 2002. PTEN in neural precursor cells: Regulation of migration, apoptosis, and proliferation. Mol. Cell Neurosci. 20: 21-29.

Ma, L., Teruya-Feldstein, J., Behrendt, N., Chen, Z., Noda, T., Hino, O., Cordon-Cardo, C., and Pandolfi, P.P. 2005. Genetic analysis of Pten and Tsc2 functional interactions in the mouse reveals asymmetrical haploinsufficiency in tumor suppression. Genes \& Dev. (this issue).

Manning, B.D. 2004. Balancing Akt with S6K: Implications for both metabolic diseases and tumorigenesis. J. Cell. Biol. 167: 399-403.

Manning, B.D., Tee, A.R., Logsdon, M.N., Blenis, J., and Cantley, L.C. 2002. Identification of the tuberous sclerosis complex-2 tumor suppressor gene product tuberin as a target of the phosphoinositide 3-kinase/Akt pathway. Mol. Cell 10: 151-162.

McManus, E.J., Collins, B.J., Ashby, P.R., Prescott, A.R., Murray-Tait, V., Armit, L.J., Arthur, J.S., and Alessi, D.R. 2004. The in vivo role of 
Manning et al.

PtdIns $(3,4,5) \mathrm{P}(3)$ binding to PDK1 PH domain defined by knockin mutation. EMBO I. 23: 2071-2082.

Neshat, M.S., Mellinghoff, I.K., Tran, C., Stiles, B., Thomas, G., Petersen, R., Frost, P., Gibbons, J.J., Wu, H., and Sawyers, C.L. 2001. Enhanced sensitivity of PTEN-deficient tumors to inhibition of FRAP/mTOR. Proc. Natl. Acad. Sci. 98: 10314-10319.

Onda, H., Lueck, A., Marks, P.W., Warren, H.B., and Kwiatkowski, D.J. 1999. $\mathrm{Tsc}^{+/-}$mice develop tumors in multiple sites that express gelsolin and are influenced by genetic background. J. Clin. Invest. 104: 687-695.

Plas, D.R. and Thompson, C.B. 2003. Akt activation promotes degradation of tuberin and FOXO3a via the proteasome. J. Biol. Chem. 278: 12361-12366.

Podsypanina, K., Ellenson, L.H., Nemes, A., Gu, J., Tamura, M., Yamada, K.M., Cordon-Cardo, C., Catoretti, G., Fisher, P.E., and Parsons, R. 1999. Mutation of Pten/Mmacl in mice causes neoplasia in multiple organ systems. Proc. Nat1. Acad. Sci. 96: 1563-1568.

Podsypanina, K., Lee, R.T., Politis, C., Hennessy, I., Crane, A., Puc, J., Neshat, M., Wang, H., Yang, L., Gibbons, J., et al. 2001. An inhibitor of mTOR reduces neoplasia and normalizes p70/S6 kinase activity in PTEN $N^{+/-}$mice. Proc. Natl. Acad. Sci. 98: 10320-10325.

Potter, C.J., Pedraza, L.G., and Xu, T. 2002. Akt regulates growth by directly phosphorylating Tsc2. Nat. Cell. Biol. 4: 658-665.

Radimerski, T., Montagne, J., Hemmings-Mieszczak, M., and Thomas, G. 2002. Lethality of Drosophila lacking TSC tumor suppressor function rescued by reducing dS6K signaling. Genes \& Dev. 16: 26272632.

Shah, O.J., Wang, Z., and Hunter, T. 2004. Inappropriate activation of the TSC/Rheb/mTOR/S6K cassette induces IRS1/2 depletion, insulin resistance, and cell survival defects. Curr. Biol. 14: 1650-1656.

Shaw, R.J., Bardeesy, N., Manning, B.D., Lopez, L., Kosmatka, M., DePinho, R.A., and Cantley, L.C. 2004. The LKB1 tumor suppressor negatively regulates mTOR signaling. Cancer Cell 6: 91-99.

Stambolic, V., Suzuki, A., delaPompa, J.L., Brothers, G.M., Mirtsos, C. Sasaki, T., Ruland, J., Penninger, J.M., Sioderovski, D.P., and Mak, T.W. 1998. Negative regulation of PKB/Akt-dependent cell survival by the tumor suppressor PTEN. Cell 95: 29-39.

Sun, H., Lesche, R., Li, D., Liliental, J., Zhang, H., Gao, J., Gavrilova, N., Mueller, B., Liu, X., and Wu, H. 1999. PTEN modulates cell cycle progression and cell survival by regulating phosphatidylinositol 3,4,5-triphosphate and Akt/protein kinase B signaling pathway. Proc. Nat1. Acad. Sci. 96: 6199-6204.

Suzuki, A., delaPompa, J.L., Stambolic, V., Elia, E.J., Sasaki, T., delBarcoBarrantes, I., Ho, A., Wakeham, A., Itie, A., Khoo, W., et al. 1998. High cancer susceptibility and embryonic lethality associated with mutation of the PTEN tumor suppressor gene in mice. Curr. Biol. 8: $1169-1178$

Tran, H., Brunet, A., Griffith, E.C., and Greenberg, M.E. 2003. The many forks in FOXO's road. Sci. STKE 2003: RE5.

Wendel, H.G., De Stanchina, E., Fridman, J.S., Malina, A., Ray, S., Kogan, S., Cordon-Cardo, C., Pelletier, J., and Lowe, S.W. 2004. Survival signalling by Akt and eIF4E in oncogenesis and cancer therapy. Nature 428: 332-337.

Zhang, H., Cicchetti, G., Onda, H., Koon, H.B., Asrican, K., Bajraszewski, N., Vazquez, F., Carpenter, C.L., and Kwiatkowski, D.J. 2003. Loss of Tsc1/Tsc2 activates mTOR and disrupts PI3K-Akt signaling through downregulation of PDGFR. J. Clin. Invest. 112: 1223-1233. 


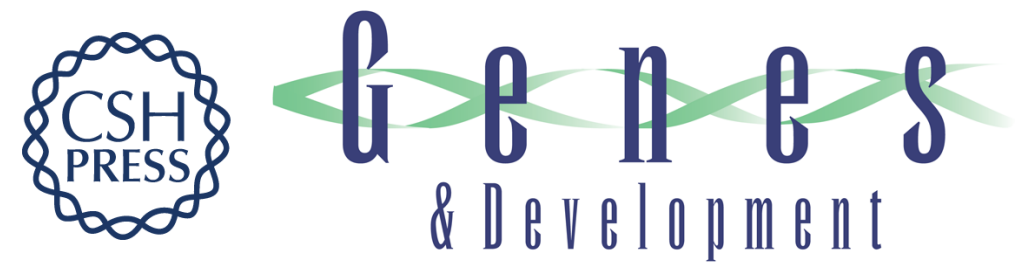

\section{Feedback inhibition of Akt signaling limits the growth of tumors lacking Tsc2}

Brendan D. Manning, M. Nicole Logsdon, Alex I. Lipovsky, et al.

Genes Dev. 2005, 19:

Access the most recent version at doi:10.1101/gad.1314605

Supplemental http://genesdev.cshlp.org/content/suppl/2005/08/03/gad.1314605.DC1
Material

References This article cites 37 articles, 17 of which can be accessed free at:

http://genesdev.cshlp.org/content/19/15/1773.full.html\#ref-list-1

License

Email Alerting Receive free email alerts when new articles cite this article - sign up in the box at the top

Service right corner of the article or click here.

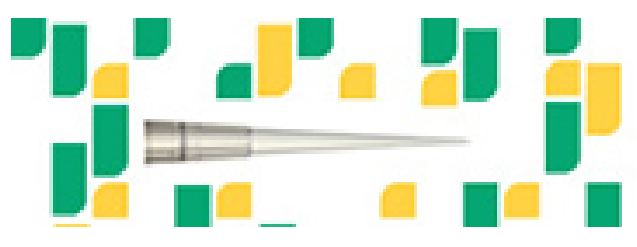

Focused on your science. 